\title{
Classificação Automática de Questões Problema de Matemática para Aplicações do Pensamento Computacional na Educação
}

\author{
Erick J. F. Costa ${ }^{1}$, Cláudio E. C. Campelo ${ }^{1}$, Lívia M. R. S. Campos ${ }^{1}$ \\ ${ }^{1}$ Universidade Federal de Campina Grande (UFCG), Paraíba, Brasil \\ Departamento de Sistemas e Computação (DSC) \\ erick.costa.ti@gmail.com, (campelo, livia)@dsc.ufcg.edu.br
}

\begin{abstract}
Recent studies indicate a greater relation of problem questions of Mathematics with Computational Thinking. However, the identification of this relation presents difficulties for a large dataset application. In this work, we present an automatic classifier of Mathematical problem questions with the aim of enabling practices involving Computational Thinking. The results show that it is possible to classify mathematical questions automatically using natural language processing and machine learning.
\end{abstract}

Resumo. Estudos recentes indicam uma maior relação de questões problema de Matemática com o Pensamento Computacional. No entanto, a identificação dessa relação apresenta dificuldades de aplicação para um grande conjunto de dados. Neste trabalho, apresentamos um classificador automático de questões problema de Matemática com o objetivo de possibilitar práticas envolvendo o Pensamento Computacional. Os resultados mostram que é possível classificar questões de Matemática de forma automática, utilizando processamento de linguagem natural e aprendizado de máquina.

\section{Introdução}

Com o avanço da tecnologia e das contribuições positivas da Ciência da Computação para a sociedade, diversas estratégias que envolvem o Pensamento Computacional (PC) foram colocadas em prática para estímulo à Resolução de Problemas (RP). O PC, de acordo com Wing (2006), é uma forma estruturada e organizada de pensar, amparada pelas competências aprendidas com a Ciência da Computação.

$\mathrm{Na}$ literatura do PC duas abordagens são fortemente exploradas: o ensino de Ciência da Computação por meio de disciplinas específicas, como programação [Costella et al. 2017]; e a introdução de conceitos da Computação em conjunto com disciplinas da Educação Básica [Barcelos et al. 2015].

Com o objetivo de apoiar aplicações práticas do PC na educação, Barr e Stephenson (2011) apresentam em seu estudo competências que são consideradas o núcleo do PC e, que devem ser estimuladas desde as séries iniciais, a saber: coleta de dados; análise de dados; representação de dados; decomposição de problemas; concepção de algoritmos; abstração de informações; automatização; simulação; e paralelização de procedimentos. 
VII Congresso Brasileiro de Informática na Educação (CBIE 2018)

Anais dos Workshops do VII Congresso Brasileiro de Informática na Educação (WCBIE 2018)

Com foco na abordagem de aplicação do PC em conjunto com disciplinas da Educação Básica e, nas competências do PC propostas por Barr e Stephenson (2011), Mestre et al. (2015) e Costa et al. (2016) apresentam um estudo da relação de conformidade de questões de Matemática com as competências do PC. Nesses estudos, foram analisadas questões do Programa Internacional de Avaliação de Estudantes (PISA) e questões tradicionalmente utilizadas em sala de aula por professores, de modo a identificar a quantidade de competências do PC estimuladas em cada questão, tal que, quanto mais competências em uma questão, maior sua conformidade ou relação com o PC. Os resultados obtidos apontam indícios de que a relação das questões com as competências propostas na literatura foi maior nas questões do PISA, visto que, o modelo de questão adotado por este exame apresentou um acumulado de até seis das nove competências do PC.

De acordo com a literatura, uma questão pode ser caracterizada como Questão Problema de Matemática (QPM) se apresentar diversas formas de resolução, diversos conteúdos e fizer alguma relação com o cotidiano [Silva and Filho 2011]. Desta maneira, foi possível observar que as questões com maior relação com o PC tendem a apresentar características de QPM, enquanto que a maior parte das questões que apresentaram uma menor relação, podem ser caracterizadas como Questão Não Problema de Matemática (QNPM).

Como destacado pelos autores, a avaliação das questões quanto à sua relação com as competências do PC foi realizada de forma manual, onde três especialistas avaliavam uma determinada questão e emitiam o parecer final de acordo com o voto da maioria. Ao considerar esse modelo de análise de relação, foi possível perceber dificuldades de aplicação para um grande conjunto de questões.

Desta forma, em face das dificuldades apresentadas na análise por voto majoritário para identificação da relação de uma questão de Matemática com o PC e, das características das questões avaliadas na literatura, neste artigo é apresentado a concepção e avaliação de um classificador probabilístico automático de QPM e QNPM.

Os resultados obtidos apontam que é possível classificar questões textuais de Matemática, em português, de forma automática e com alta capacidade de predição. Em um contexto mais amplo, o classificador será utilizado em um ambiente educacional (em software) que permita estimular o PC e aprimorar a capacidade de RP em estudantes do Ensino Básico na disciplina de Matemática.

O restante deste artigo está estruturado da seguinte forma: na Seção 2 é apresentada a fundamentação teórica e os trabalhos relacionados; na Seção 3 são discutidos os procedimentos metodológicos; na Seção 4, os resultados são apresentados; a Seção 5 conclui o artigo e aponta os trabalhos futuros.

\section{Fundamentação Teórica}

O PC pode ser considerado uma estratégia para RP assim como a Matemática. No entanto, algumas características precisam ser consideradas na Matemática, para estimular à RP, principalmente no formato como seus exercícios/questões são apresentados.

A literatura destaca que, para uma questão de Matemática ser considerada uma questão problema e estimular à RP, ela deve apresentar algumas características importan- 
VII Congresso Brasileiro de Informática na Educação (CBIE 2018)

Anais dos Workshops do VII Congresso Brasileiro de Informática na Educação (WCBIE 2018)

tes e, a principal delas, é a contextualização. Desta maneira, faz-se necessário que os envolvidos no processo de resolução de uma questão de Matemática relacionem o cotidiano com a aplicação dos conteúdos aprendidos [Krulik and Reys 1997].

Santos (1997) descreve algumas estratégias para estimular à RP na Matemática, além disso, destaca a importância da RP para a construção do entendimento da Matemática e seu papel na sociedade. As principais estratégias apresentadas pela autora são: buscar padrões; usar deduções; fazer generalizações; trabalhar com correlações; e construir gráficos.

Ao observar as estratégias propostas por Santos (1997) e realizar um paralelo com as competências definidas por Barr e Stephenson (2011) para estimular o PC, podemos destacar a existência de uma relação entre as definições apresentadas. Se tomarmos como exemplo a competência "representação de dados", podemos facilmente relacioná-la com a estratégia "construção de gráficos". O mesmo pode ser observado para a competência do PC "abstração de informações", relacionada com as estratégias dedutivas e a realização de correlações.

De maneira prática, ao considerar os estudos realizados por Mestre et al. (2015) e Costa et al. (2016), podemos observar que as questões do PISA podem ser classificadas como QPM e, as questões tradicionalmente utilizadas em sala de aula por professores, como QNPM. Na Figura 1 a) e 1 b) são exemplificadas essas questões.

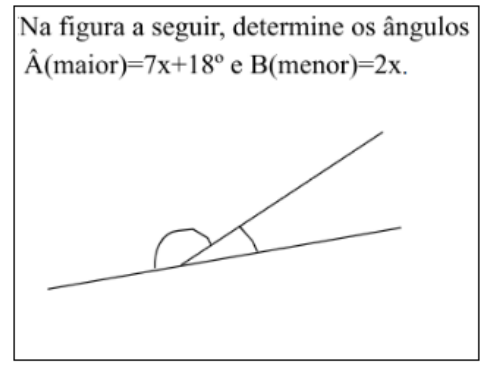

a)
O número mensal de passagens de uma determinada empresa aérea aumentou no ano passado nas seguintes condiçőes: em janeiro foram vendidas 33000 passagens; em fevereiro, 34 500; em março, 36 000. Esse padrão de crescimento se mantém para os meses subsequentes. Quantas passagens foram vendidas por essa empresa em julho do ano passado?

A 38000

B 40500

C 41000

(D) 42000 G 48000

Figura 1. a) Exemplo de QNPM. b) Exemplo de QPM.

Ao observar os exemplos, percebemos que as questões tradicionais são estruturadas para colocar em prática o conhecimento adquirido em um determinado conteúdo e apresentam um formato de resolução bem definido. Por outro lado, as questões do PISA apresentam um caráter mais interpretativo e não estão focadas em um único processo de resolução. Estes aspectos demonstram a diferença entre uma QPM e uma QNPM, além disso, apontam também a sua relação com as competências do PC, maior nas questões do PISA (seis de nove competências) e menor nas questões tradicionais (quatro de nove competências).

Ao considerar o PC de maneira conjunta com disciplinas do Ensino Básico, como a Matemática, podemos observar a abordagem proposta por Marques et al. (2017). Neste estudo, os autores apresentam um modelo conceitual para estimular o PC na Matemática baseado na Taxonomia de Bloom. O modelo coloca em prática a elaboração e resolução de questões que permitem o aprendizado de conceitos matemáticos e habilidades do PC. Como resultados, foram apresentadas questões elaboradas de acordo com o modelo proposto pelos autores. A Figura 2 apresenta uma questão produzida de acordo com a pro- 
VII Congresso Brasileiro de Informática na Educação (CBIE 2018)

Anais dos Workshops do VII Congresso Brasileiro de Informática na Educação (WCBIE 2018)

posta.

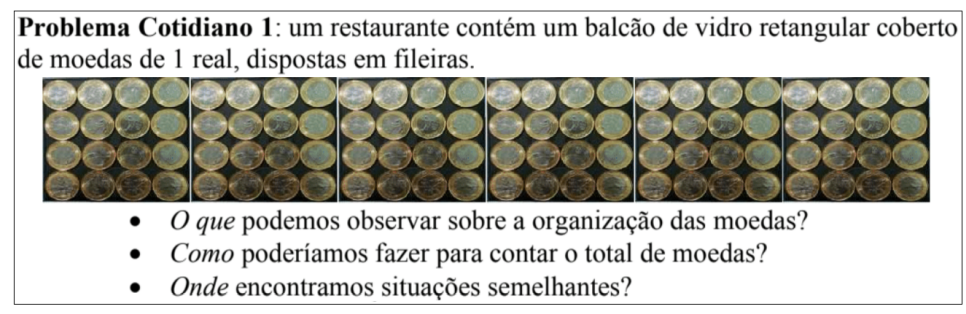

Figura 2. Exemplo de questão produzida em Marques et al. (2017).

Ao observar o modelo de questão produzido por Marques et al. (2017), podemos destacar a semelhança estrutural dessas questões com as questões do PISA, avaliadas por Mestre et al. (2017). Essas semelhanças apontam para uma maior relação com as competências do PC, diferente das questões avaliadas por Costa et al. (2016), que tendem a apresentar características de QNPM e uma menor relação com essas competências.

\subsection{Trabalhos Relacionados}

A análise de textos de forma automática tem apresentado avanços em ambientes educacionais, como destacado por Dionísio et al. (2017). Em seu estudo, os autores apresentam um classificador automático de colaborações em ambientes virtuais de ensino, onde seu objetivo é auxiliar os professores no acompanhamento das postagens e incentivar os alunos. Os resultados obtidos pelos autores apontam que, ao considerar o cenário de avaliação apresentado, é possível identificar colaborações para diferentes classes características.

Em Ramos et al. (2017), os autores apresentam em seu estudo estratégias para detectar evasão de alunos em cursos à distância. Como resultados, utilizando técnicas de aprendizagem supervisionada, os autores apontam que é possível detectar evasão em ambientes de ensino à distância com uma alta capacidade de predição ao utilizar um modelo de regressão linear.

Alguns estudos apresentam experimentos voltados para a geração de questões de forma automática [Azevedo et al. 2017]. No contexto de classificação de questões, é possível identificar estudos que consideram diferentes níveis de dificuldade, como apresentado em Zhang e Lee (2003).

O estudo aqui apresentado, discute uma forma diferente de conduzir os procedimentos de estímulo à RP por meio do PC na Matemática, ao propor que a classificação de questões seja realizada de forma automatizada, mais especificamente, considerando uma QPM e uma QNPM.

\section{Metodologia}

Para guiar o estudo aqui apresentado, a seguinte Questão de Pesquisa (QP) foi definida:

- QP: É possível classificar de forma automática questões textuais de Matemática, em português, entre uma QPM e uma QNPM ao utilizar técnicas de processamento de linguagem natural e aprendizado de máquina?

Para responder a QP, as seguintes Hipóteses $(\mathrm{H})$ foram definidas: 
VII Congresso Brasileiro de Informática na Educação (CBIE 2018)

Anais dos Workshops do VII Congresso Brasileiro de Informática na Educação (WCBIE 2018)

- H0: Não é possível classificar de forma automática questões textuais de Matemática, em português, entre uma QPM e uma QNPM ao utilizar técnicas de processamento de linguagem natural e aprendizado de máquina.

- H1: É possível classificar de forma automática questões textuais de Matemática, em português, entre uma QPM e uma QNPM ao utilizar técnicas de processamento de linguagem natural e aprendizado de máquina, mas com baixa capacidade de predição.

- H2: É possível classificar de forma automática questões textuais de Matemática, em português, entre uma QPM e uma QNPM ao utilizar técnicas de processamento de linguagem natural e aprendizado de máquina, mas com alta capacidade de predição.

As métricas usadas para verificar as hipóteses e responder a questão de pesquisa foram:

- Precisão (P): é o número de classificações corretas, ou seja, das questões que foram classificadas como QPM, quantas efetivamente eram QPM?. A fórmula para calcular a métrica $P$ pode ser observada na Equação 1;

$$
P=\frac{\text { VerdadeirosPositivos }}{\text { VerdadeirosPositivos }+ \text { FalsosPositivos }}
$$

- Cobertura (C): é a frequência de classificações corretas, ou seja, das questões que são QPM, quão frequente foram classificadas como QPM?. A fórmula para calcular a métrica $\mathrm{C}$ pode ser observada na Equação 2;

$$
C=\frac{\text { VerdadeirosPositivos }}{\text { VerdadeirosPositivos }+ \text { FalsosNegativos }}
$$

- e Medida F (F1): é a combinação da Precisão e da Cobertura, indicando a qualidade geral do classificador. A fórmula para calcular a métrica F1 pode ser observada na Equação 3.

$$
F 1=2 \cdot \frac{P \cdot C}{P+C}
$$

Essas métricas são comumente utilizadas para avaliação de preditores em aprendizado de máquina [Baeza and Ribeiro 2013]. Além delas, também foram consideradas a análise da curva de aprendizagem, que indica o nível com que se aprende uma determinada tarefa à medida com que se conhece mais informações sobre a mesma e, a curva característica da operação do receptor (curva ROC), que ilustra o desempenho de um sistema classificador binário.

\subsection{Banco de Questões}

Para que fosse possível conceber o classificador, inicialmente foi organizado um banco de questões para ser usado como treinamento e teste. Primeiramente, foram selecionadas questões caracterizadas como QPM, questões do PISA e do Exame Nacional do Ensino Médio (ENEM).

O segundo passo foi selecionar questões caracterizadas como QNPM. Para compor esta parte da amostra, foram coletadas questões em escolas públicas e particulares 
VII Congresso Brasileiro de Informática na Educação (CBIE 2018)

Anais dos Workshops do VII Congresso Brasileiro de Informática na Educação (WCBIE 2018)

do município de Campina Grande - PB e de repositórios de questões online (Brainly ${ }^{1}$, Mundo Educação e Matemáticoteca ${ }^{3}$ ).

Além das amostras descritas até então, também foram consideradas as questões disponibilizadas pelo Sistema de Avaliação de Rendimento Escolar do Estado de São Paulo (SARESP). Esse repositório apresenta questões dos dois tipos (QPM e QNPM). Neste caso, foi necessário realizar a separação das questões e, para tal, tomou-se como base a experiência em trabalhos anteriores no mesmo contexto e as definições para QPM e QNPM apresentadas na literatura [Mestre et al. 2015, Costa et al. 2016, Marques et al. 2017, Santos 1997].

Ao agrupar as questões da amostra, obteve-se um total de 312 questões QPM e 314 questões QNPM, um total geral de 626 questões.

O último passo desta etapa foi a montagem do conjunto de dados, onde para cada questão, foram extraídos os enunciados que compõem o texto a ser avaliado pelo classificador. Após a realização da extração dos enunciados, foram inseridos os rótulos com a classe de identificação desejada para cada enunciado de questão de acordo com seu grupo.

\subsection{Concepção e Validação do Classificador}

O classificador de questões foi escrito em Python $^{4}$ e foi utilizado um modelo probabilístico baseado no teorema de Bayes com distribuição multinominal (Multinomial Naive Bayes). Esse modelo foi escolhido por seu caráter simples e desempenho satisfatório para uma quantidade reduzida de dados.

Para avaliar o classificador, foram utilizadas diferentes configurações para entender qual delas se adequava melhor ao problema. Foram utilizadas duas abordagens para bag-of-words (contagem de termos): uma a count vectorizer e a outra TF-IDF vectorizer. A diferença principal entre as duas abordagens de contagem está no formato de análise dos documentos, onde o count vectorizer converte todo o documento de texto em uma matriz que contém o peso das identificações dos termos, enquanto que, o TF-IDF vectorizer faz uma análise mais apurada para atribuir os pesos aos termos, analisando não somente a presença em grande quantidade, mas se ele está presente também na maioria dos textos do conjunto de dados [Brink et al. 2017].

Além da definição das abordagens de contagem, também foi considerada a variação de n-grama (combinação de termos) de 1 (termos únicos) até 2 (combinação de até dois termos em sequência), além disso, foi definido um mínimo padrão e a variação do máximo, pois em testes iniciais ao considerar apenas bigramas, observou-se uma queda brusca na capacidade de predição do classificador [Elberrichi and Aljohar 2007].

A última configuração considerada nos testes para aferição da capacidade de predição do classificador foi a taxa de descarte de termos. Para este estudo, foram considerados testes com descarte de termos muito frequentes (pesos acima de $75 \%$ ) para as duas abordagens de contagem, além disso, também foram realizados testes sem descarte. $\mathrm{O}$ descarte foi considerado, pois pesos muito altos indicam termos poucos discriminatórios

\footnotetext{
${ }^{1}$ Brainly: https://brainly.com.br/

${ }^{2}$ Mundo Educação: https://mundoeducacao.bol.uol.com.br/

${ }^{3}$ Matemáticoteca: http://www.matematicoteca.com/

${ }^{4}$ Scikit learn: http://scikit-learn.org/stable/index.html
} 
VII Congresso Brasileiro de Informática na Educação (CBIE 2018)

Anais dos Workshops do VII Congresso Brasileiro de Informática na Educação (WCBIE 2018)

para as classes, no caso deste estudo, QPM e QNPM.

Por fim, os dados coletados para as configurações do classificador (P, C e F1), foram as medianas da validação cruzada (cross-validator) com divisão dos dados de forma estratificada $(k$-folds $=10)$ para treinamento e teste [Pedregosa et al. 2011].

\section{Resultados}

Os resultados dos testes ao seguir a proposta metodológica de avaliação podem ser observados na Tabela 1. Esses resultados apontam que, dentre todas as configurações avaliadas, a que apresentou melhor mediana para as métricas definidas foi a configuração T1.75. Essa configuração leva em consideração para quantificação dos termos o $T F-I D F$, a variação de n-gramas para apenas um termo e o descarte de termos com pesos acima de 0.75 .

Tabela 1. Resultados dos testes ao considerar Precisão, Cobertura e Medida F.

\begin{tabular}{|l|l|l|l|l|l|l|}
\hline Contagem de Termos & N-grama & Descarte & Título & Precisão & Cobertura & Medida F \\
\hline Count & $(\min =1, \max =1)$ & Acima de 0.75 & $\mathrm{C} 1.75$ & 0.88 & 0.85 & 0.85 \\
\hline Count & $(\min =1, \max =1)$ & Sem descarte & $\mathrm{C} 1.00$ & 0.86 & 0.83 & 0.83 \\
\hline Count & $(\min =1, \max =2)$ & Acima de 0.75 & $\mathrm{C} 2.75$ & 0.81 & 0.73 & 0.71 \\
\hline Count & $(\min =1, \max =2)$ & Sem descarte & $\mathrm{C} 2.00$ & 0.82 & 0.73 & 0.70 \\
\hline TF-IDF & $(\min =1, \max =1)$ & Acima de 0.75 & $\mathrm{~T} 1.75$ & 0.92 & 0.90 & 0.91 \\
\hline TF-IDF & $(\min =1, \max =1)$ & Sem descarte & $\mathrm{T} 1.00$ & 0.90 & 0.89 & 0.89 \\
\hline TF-IDF & $(\min =1, \max =2)$ & Acima de 0.75 & $\mathrm{~T} 2.75$ & 0.90 & 0.88 & 0.88 \\
\hline TF-IDF & $(\min =1, \max =2)$ & Sem descarte & $\mathrm{T} 2.00$ & 0.88 & 0.85 & 0.85 \\
\hline
\end{tabular}

Com o objetivo de identificar o viés e a variação do classificador de questões para a configuração T1.75, foi analisada a curva de aprendizagem. Neste procedimento, a técnica de validação cruzada (cross-validator) com divisão dos dados de maneira aleatória $($ shuffle split $=10)$ foi considerada e o resultado pode ser observado na Figura 3.

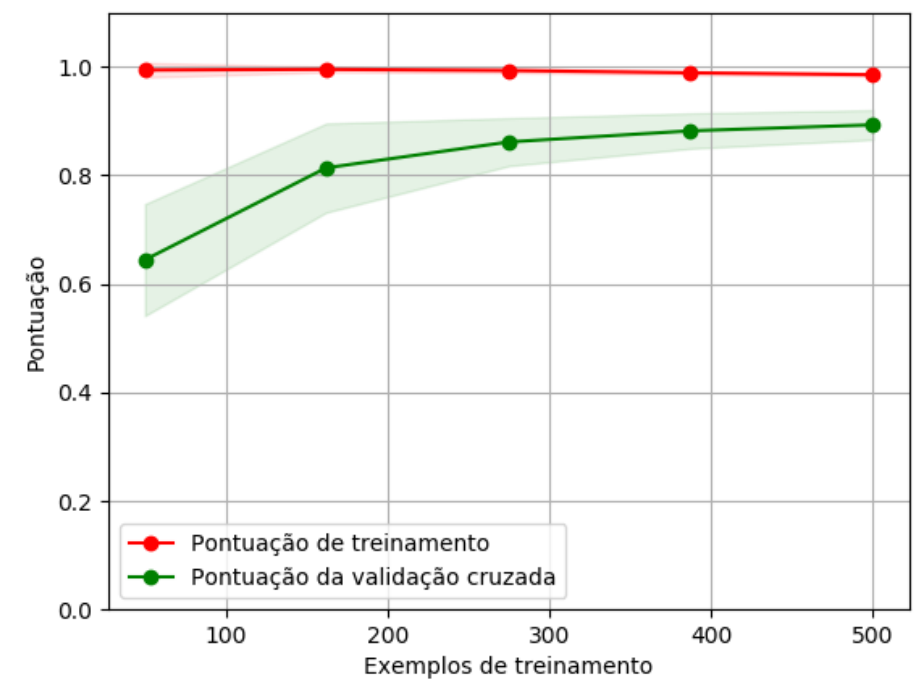

Figura 3. Curva de aprendizagem para o classificador de questões. 
VII Congresso Brasileiro de Informática na Educação (CBIE 2018)

Anais dos Workshops do VII Congresso Brasileiro de Informática na Educação (WCBIE 2018)

Ao analisar a curva de aprendizagem, foi possível observar um baixo viés e variação, pois a medida que se aumenta a amostra de treinamento, a pontuação da amostra de testes cresce. Estes resultados indicam uma alta capacidade de generalização por parte do modelo usado no classificador de questões.

A Figura 4 apresenta a taxa de verdadeiros positivos e falsos positivos indicadas por meio de uma curva ROC (Receiving Operating Characteristic) para a configuração T1.75. Este procedimento tem por objetivo medir o equilíbrio do classificador em relação as suas predições.

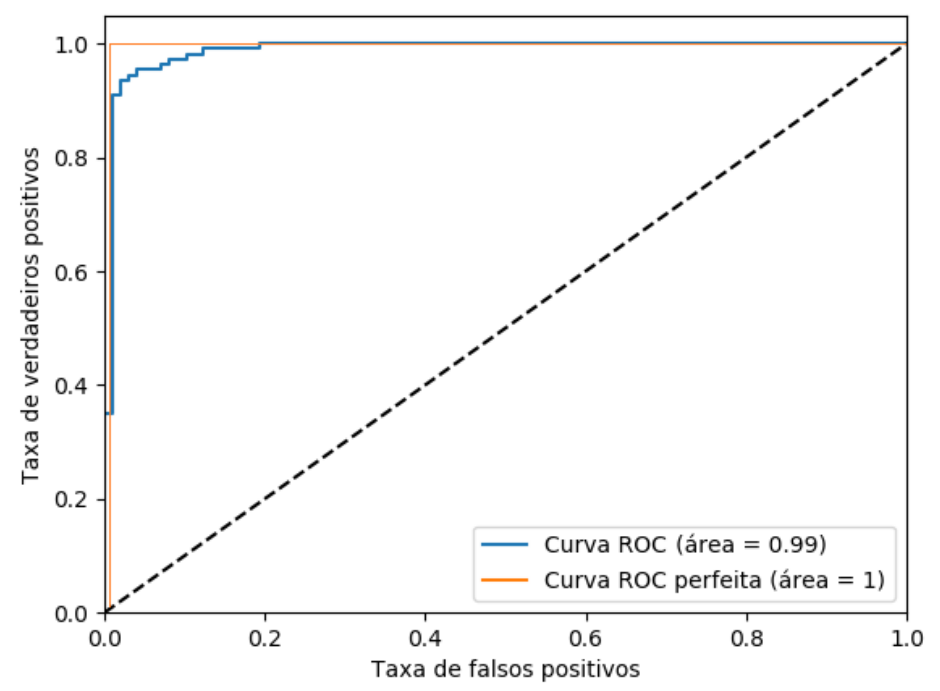

Figura 4. ROC curva que indica a confiança do classificador de questões.

Ao observar a curva gerada, foi possível identificar que ela apresenta uma disposição convexa (hump sheped) com tendência ao topo esquerdo do gráfico. A perfeição do classificador seria observada no caso de a curva ter se comportado como a indicação em laranja e, embora a configuração T1.75 não tenha apresentado $100 \%$ de predições corretas, foi possível identificar que ele apresenta uma alta taxa de confiança, elevando a taxa de Cobertura e reduzindo a quantidade de erros de classificação.

Diante dos resultados apresentados e ao analisar as métricas de avaliação definidas na metodologia deste trabalho, podemos rejeitar as hipóteses $\mathrm{H} 0$ e $\mathrm{H} 1$, visto que, foi possível classificar questões textuais de Matemática, em português, de forma automática e obter uma alta capacidade de predição. Logo, podemos aceitar H2 e responder a QP definida na metodologia de maneira positiva.

\section{Conclusão e Trabalhos Futuros}

Estudos indicam que questões de Matemática com maior relação com o PC são caracterizadas como QPM e as que apresentam uma menor relação como QNPM. Nesses estudos, o modelo de avaliação das questões foi baseado em votação majoritária, o que dificulta sua aplicação para um grande conjunto de questões. Desta maneira, neste artigo foi apresentado um classificador automático de QPM e QNPM para viabilizar aplicações do PC na educação e estimular a capacidade de RP. 
VII Congresso Brasileiro de Informática na Educação (CBIE 2018)

Anais dos Workshops do VII Congresso Brasileiro de Informática na Educação (WCBIE 2018)

Os resultados mostram que foi possível classificar questões textuais de Matemática entre QPM e QNPM com alta Precisão, Cobertura e Medida F. Além de identificar uma alta confiabilidade e capacidade de generalização do modelo de classificação.

O classificador irá contribuir para estudos futuros que tem por objetivo automatizar o processo de elaboração, classificação e resolução de questões de Matemática em maior conformidade com PC. Além disso, possibilitará estudos mais detalhados em relação ao impacto dessas questões na capacidade de RP.

Em um contexto mais amplo, como apresentado na introdução deste trabalho, o classificador será utilizado em um ambiente educacional (em software) que permita estimular o PC e aprimorar a capacidade de RP em estudantes do Ensino Básico. Nesse ambiente, será possível medir o tempo de resposta para uma questão, as questões mais acertadas, o desempenho de cada aluno, entre outras características que não são identificadas em ambientes menos controlados.

Como trabalhos futuros, pretende-se aprimorar a análise das questões usadas para treinamento e teste do classificador e sua relação com as competências do PC. Além disso, possibilitar a análise das questões por especialistas e identificar possíveis ruídos nas classes usadas na rotulação dos enunciados. Após esta etapa, planeja-se conceber um classificador que seja capaz não só de predizer QPM e QNPM, mas também identificar quais competências do PC, especificamente, são abordadas nas questões.

No que diz respeito ao classificador concebido a avaliando neste trabalho, é de grande importância a realização de testes envolvendo outros algoritmos de classificação.

\section{Referências}

Azevedo, D., Ferreira, R., Mendonca, V., and Miranda, P. (2017). Aplicação de análise de sentimento em fóruns educacionais para prevenir evasão. In Brazilian Symposium on Computers in Education (Simpósio Brasileiro de Informática na Educação-SBIE), volume 28, page 1097.

Baeza, Y. R. and Ribeiro, N. B. (2013). Recuperação de Informação: Conceitos e Tecnologia das Máquinas de Busca. Bookman Editora.

Barcelos, T., Muñoz, R., Acevedo, R. V., and Silveira, I. F. (2015). Relaçoes entre o pensamento computacional e a matemática: uma revisao sistemática da literatura. In Anais dos Workshops do Congresso Brasileiro de Informática na Educação, volume 4, page 1369.

Barr, V. and Stephenson, C. (2011). Bringing computational thinking to k-12: what is involved and what is the role of the computer science education community? Acm Inroads, 2(1):48-54.

Brink, H., Richards, J. W., and Fetherolf, M. (2017). Real-world machine learning. Manning.

Costa, E. J. F., Sampaio, L., and Guerrero, D. (2016). Pensamento computacional na educação básica: Uma análise da relação de questões de matemática com as competências do pensamento computacional. In Anais dos Workshops do Congresso Brasileiro de Informática na Educação, volume 5, page 1060. 
VII Congresso Brasileiro de Informática na Educação (CBIE 2018)

Anais dos Workshops do VII Congresso Brasileiro de Informática na Educação (WCBIE 2018)

Costella, L., Trentin, M., Amarante, V., and Teixeira, A. (2017). Construção de ambiente de ensino de robótica remota: Democratizando o desenvolvimento do pensamento computacional em alunos da educação básica. In Brazilian Symposium on Computers in Education (Simpósio Brasileiro de Informática na Educação-SBIE), volume 28, page 354.

Dionísio, M., Ferreira, R., Cavalcanti, A., Carvalho, R., and Neto, S. (2017). Mineração de texto aplicada à identificação de colaboração em fóruns educacionais. In Brazilian Symposium on Computers in Education (Simpósio Brasileiro de Informática na Educação-SBIE), volume 28, page 1437.

Elberrichi, Z. and Aljohar, B. (2007). N-grams in texts categorization. Scientific Journal of King Faisal University (Basic and Applied Sciences), 8(2):1428H.

Krulik, S. and Reys, R. E. (1997). A resolução de problemas na matemática escolar. Ed. Atual.

Marques, M., Cavalheiro, S., Foss, L., Avila, C., and Bordini, A. (2017). Uma proposta para o desenvolvimento do pensamento computacional integrado ao ensino de matemática. In Brazilian Symposium on Computers in Education (Simpósio Brasileiro de Informática na Educação-SBIE), volume 28, page 314.

Mestre, P., Andrade, W., Guerrero, D., Sampaio, L., da Silva Rodrigues, R., and Costa, E. (2015). Pensamento computacional: Um estudo empírico sobre as questões de matemática do pisa. In Anais dos Workshops do Congresso Brasileiro de Informática na Educação, volume 4, page 1281.

Pedregosa, F., Varoquaux, G., Gramfort, A., Michel, V., Thirion, B., Grisel, O., Blondel, M., Prettenhofer, P., Weiss, R., Dubourg, V., et al. (2011). Scikit-learn: Machine learning in python. Journal of machine learning research, 12(Oct):2825-2830.

Ramos, J. L. C., Gomes, A. S., Rodrigues, R., Silva, J., de Souza, F. d. F., de Gouveia Zambom, E., and Prado, L. (2017). Um modelo preditivo da evasão dos alunos na ead a partir dos construtos da teoria da distância transacional. In Brazilian Symposium on Computers in Education (Simpósio Brasileiro de Informática na Educação-SBIE), volume 28, page 1227.

Santos, V. d. (1997). Avaliação de aprendizagem e raciocínio em matemática: métodos alternativos. Rio de Janeiro: Instituto de Matemática da UFRJ-Projeto Fundão.

Silva, C. M. S. and Filho, M. G. S. (2011). Matemática: Resolução de Problemas, volume 1. Liber Livro.

Wing, J. M. (2006). Computational thinking. Communications of the ACM, 49(3):33-35.

Zhang, D. and Lee, W. S. (2003). Question classification using support vector machines. In Proceedings of the 26th annual international ACM SIGIR conference on Research and development in informaion retrieval, pages 26-32. ACM. 